\title{
Adaptive Domotic System in Green Buildings
}

\author{
Diego Rodríguez-Gracia, José A. Piedra-Fernández and Luís Iribarne \\ Department of Informatics \\ University of Almería \\ Ctra. Sacramento s/n \\ Email: http://acg.ual.es/\#Members
}

\begin{abstract}
This paper presents an adaptive domotic system in green buildings. In our case, the data of sensor and devices were controlled in CIESOL center. The adaptive domotic system uses a Fuzzy Lattice Reasoning classifier for predicting building energy performance depending on the user condition. Training and testing of classifiers were carried out with temperature condition data acquired for $\mathbf{4}$ months (February, May, July and November) in the case building called CIESOL. The results show a hihg accuracy rates with a mean absolute error between $0 \%$ and $0.21 \%$.
\end{abstract}

Keywords-fuzzy lattice reasoning; supervised clasification; adaptive domotic system; smart building; energy efficiency.

\section{INTRODUCTION}

In this paper we present a specific part of the project titled Evolving dynamic systems in the cloud: A framework toward the smart user interfaces TIN2013-41576-R. This project aims to develop a schema for the adaptation of dynamic computer systems at run time (i.e., those modifying their structure in time) by using interdisciplinary techniques of model-driven engineering, cloud computing and soft computing. Componentbased computer systems are traditionally determined at design time according to a component architecture and to starting requirements. It would be useful that in dynamic computer systems with different external elements such as the user's interaction, profiles, collaboration or environment, among others, the implicit architecture changes at run time to adjust to the environment's new needs. This project will focus on the Cloud Computing management and pursue research in Model-as-a-Service (MaaS). We will also develop a natural interface model as a human-computer interaction mechanism based on natural communication, providing the system with a new set of input variables that will influence on the process analysis for the evolution. By including this new model of natural interaction, we pursue a step forward in the research towards the Smart User Interfaces. Here, user interface refers to the communication method between the person and the environment or system like, for instance, a graphical user interface. One advantage of this proposal is that it abstracts and isolates the entire evolution process of the computer system (being in the Cloud) as this is independent from the platform. This proposal can directly be applied in certain dynamic computer systems of social character, such as domotics, intelligent buildings, robotics, graphical interface users, etc. We will work in two real world scenarios for the experimentation of the evolution process of dynamic systems. One is in the domain of component-based user interfaces, applied to the environmental management. In order to compare and validate the proposal, we will develop another scenario in the scope of social well-being applied to domotics for the comforts of home. Both this new scenario and the project are aimed to make easier the quality of life of disabled people and people in general. The project is aligned with the challenges of Spanish Strategic Action Digital Economy and Society of CICYT-I 2013-2020.

In particular, this paper develops a learning system that collects information from the user interaction with the system and generates the corresponding rules that will define the knowledge of the system. These rules will be the ones used by the adaptive system to carry out the corresponding transformations at runtime, achieving the adaptation of system to the user. In the case study, we have developed a learning system capable of discerning the comfort temperatures of users or user groups of the CIESOL [1] building.

Our proposal is based on the schema for the dynamic adaptation of computer systems at run time [2] adding the novelty that the rules that will define the adaptation process are not static and preset but can be changed over time by the decision making system as a result of the interaction between the users and the system, hereby even creating new adaptation rules if necessary.

Fig. 1 shows the schema of the adaptive domotic system of our proposal, where the decision making system will generate new adaptation rules from the registration through time of the states of the sensors and actuators of the domotic system. These states are defined based on the interaction between the user and the domotic system. These adaptation rules will afterwards be transformed into control rules and added to the system control by the adaptation process.

Actually, the home automation for energy efficiency is a relevant research area. Our study refers to electronic environments that are sensitive and responsive to the presence of people. This paper introduces several aspects of intelligent building and energy efficient. The most important problem is to control the energy consumption. Our idea is to help during the accurate estimation of energy consumption in green buildings. For this purpose, it is useful the integration of artificial intelligence with building services systems.

Some studies found in the literature related about the energy consumption and interior comfort level of the low-energy house, one of them is [3] in the campus of POLITEHNICA University of Bucharest. They implemented Smart Building Controller with an execution mechanism driven by a set of policies, which activates the heating system. Other studies were focusing on implementation of the generic Human-Robot Cloud architecture [4] in the Smart Buildings scenario: a realworld application with immediate benefits in energy optimization and energy savings. In [4] some sensors with a number 
of processing and actuation elements, such as including face detection and people trackers, are transformed to a prototypical reconfigurable distributed extended cognitive system, which can support multiple applications in the future.

About the methodologies used for controlling smart buildings, some of the methodologies are broadly based on defining simple logical inferences using the building different measurement sensors and the presence sensors. [5], [6], [7]. Other methodologies choose to predict users pattern of behavior using Bayesian inference techniques (Bayesian network) [8]. In accordance with using Bayesian network to predict users behavior in [9] is proposed a method to predict energy consumption by inhabitant. On the other hand, iDorm [10] is presented here as an adaptive system (able to learn from user interaction and to predict future needs) for embedded agents. These agents use fuzzy-logic-based incremental synchronous learning (ISL). Finally in [11] the use of Markov Chains is proposed in order to establish the occupation probability for certain areas. A comparative study of the different methodologies and technologies used for controlling smart buildings is found in [12]

The goal is to develop an adaptive domotic system that allows to control the energy consumption in CIESOL. For this purpose, we implemented a Fuzzy Lattice Reasoning system to determine the user demand of temperature per each room in this building. One of the main advantage is the skill to recognize an user type depend on the data acquisition from indoor and outdoor sensors.

The layout of this paper is as follows. Section 2 presents a case of study based on green building including acquisition and preprocessing. Section 3 describes the main concept of fuzzy lattice reasoning classifier. Section 4 show the experimental results including discussions. The conclusion draw and future work are shown in Section 5.

\section{CASE OF STUDY: GREEN BUILDING}

\section{A. Solar Energy Research Center}

Solar Energy Research Center (CIESOL) is a joint research establishment, founded and managed according to an agreement signed in April 2005 between the University of Almería and the Centre for Energy, Environment and Technology (CIEMAT) at the Ministry of Economy and Competitiveness. The center is located at the University of Almeria. It was built with the support of the PSE-ARFRISOL project (The Singular Strategic Project - Bioclimatic Architecture and Solar Cooling), PS-120000-2005-1, financed by the Spanish Ministry of Innovation and Science and coffinanced by the FEDER fund for 7 years (2005-2012). CIESOL [1] was built using bioclimatic standards and its design is aimed at efficient energy use. CIESOL [1] engages in research and technology transfer activities in the field of solar energy applications concerning: organometallic photochemistry, water treatment, environmental chemistry, modeling and automatic control of solar systems, home automation for energy efficiency, as well as solar cooling and solar resources assessment.

\section{B. Data Acquisition and Preprocessing}

CIESOL [1] (single-store building) comprises an area of $1100 \mathrm{~m} 2$ with 10 laboratories, 5 offices and a conference

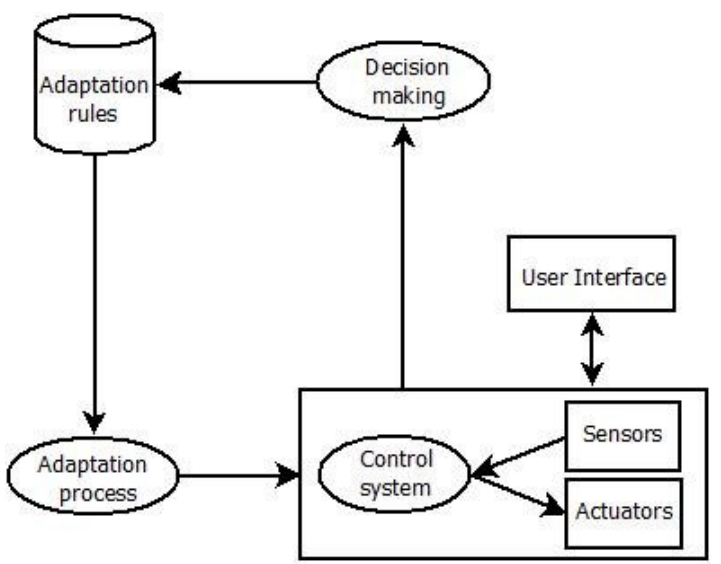

Fig. 1. Adaptive domotic system schema.

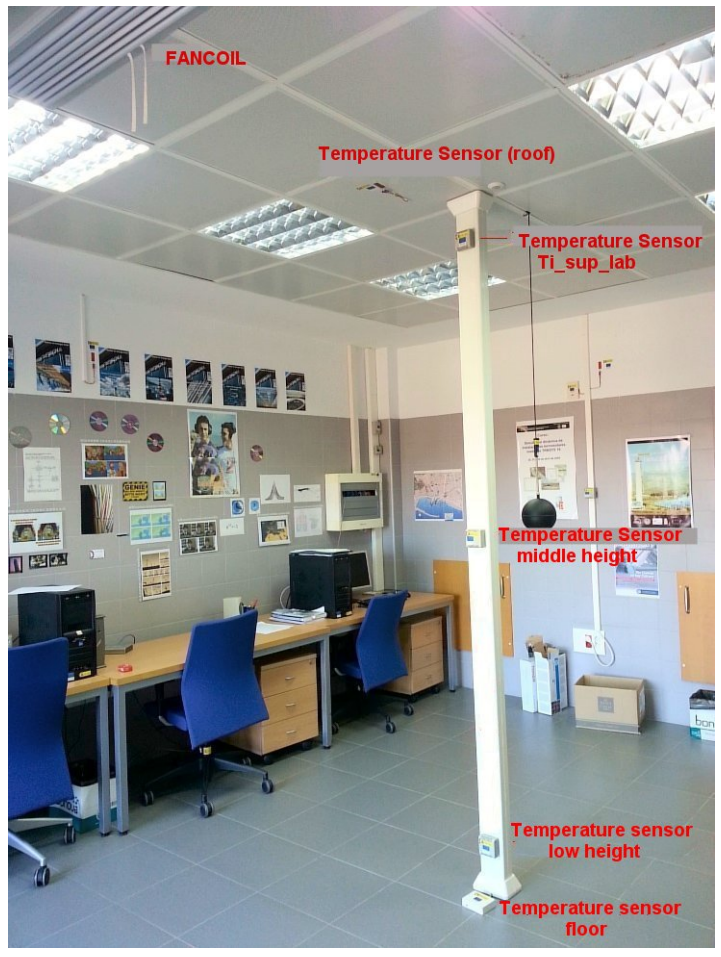

Fig. 2. Laboratory 8: the location of temperature and fancoil sensors.

room with a maximum occupancy higher than 75 people. This building uses a weather station with outdoor and indoor sensors. The data from different sensors are parameterized and colleted every minute for 24 hours a day, 365 days a year. The main environmental values are temperature, humidity, radiation and wind speed.

In our proposal, the data set are colleted by the temperature and the motion sensors located inside the rooms of the building during four months in 2014. February, May, July and October are most representative months for each season (in regard to the temperature values in the province of Almería with the highest human activity in different rooms located in CIESOL. The Fig. 2 shows the location of the fancoil and temperature 


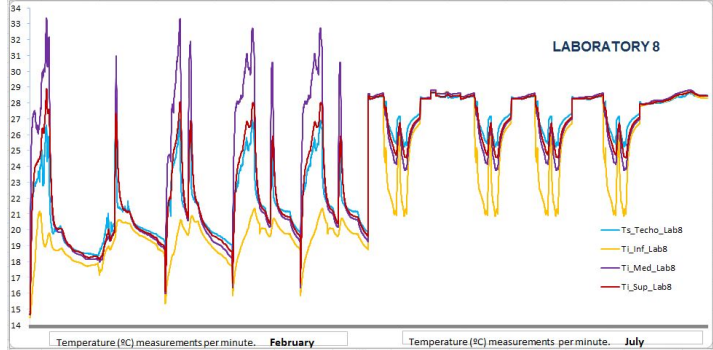

Fig. 3. Laboratory 8: temperature measurements.

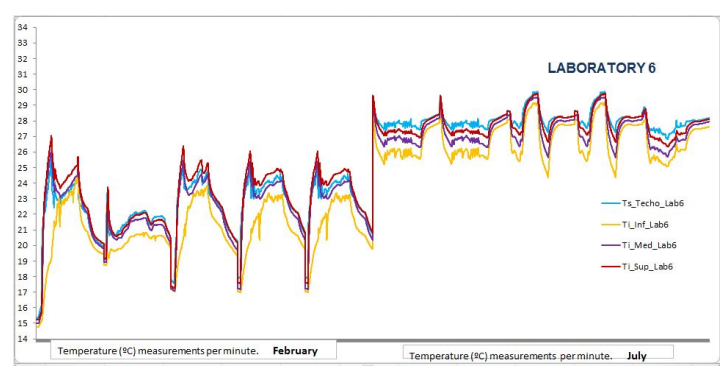

Fig. 4. Laboratory 6: temperature measurements.

sensors in one of the rooms that was studied.

From the study of the data collected was deduced, that the measures during business hours (from Monday to Friday, from 8:00 am to 20:00 pm hours) in several rooms varied significantly, demonstrating the desired temperature or comfort temperature depends on preferences of users and is not a priori known value. In Fig. 3 and 4 shows the temperatures measured by the four temperature sensors located in two rooms of the CIESOL building in which we have focused this study. The roof-sensor values are not shown because this sensor collects fancoil temperature output, and not the temperature of the room. These measurements correspond to the same period of time (second week of February and July, Monday to Friday) in each room. The differences between the temperatures of the rooms can be appreciated when there is human presence during working hours. For this reason, the difference between the desired temperature depending on the user preferences.

After data were preprocessed, five variables were recognized as the relevant variables to define the state of the fan coil (three state: OFF, heating and cooling) in the various rooms studied, resulting in a total of $60 \times 24 \times 121=174,200$ data vectors per room, of which $4.638(2.66 \%)$ are not usable by the inconsistency of some value (had at least a missing value). The five variables where we have focused our study are shown in TableI.

The AlarActivePres variable indicates whether the central building alarm CIESOL is activated or not activated. If so (AlarPresActiva $=1$ ) then fan coil have to be off (FANCLab $=0$ ). The same idea is applied to the motion sensor of the rooms. In the event that nobody is in the room (SenMovLab $=0$ ) the fan coil have to also be turned off without regard to the measured temperature in the room (Ti_Sup_Lab).

During the preprocessing that was performed on the data of study, the time variable measuring the temperature of the rooms (date:hour:minutes format) was processed and a new variable called Month was created. This new variable indicates the month the which were performed measurements with sensors of different rooms (February $=1$, May $=2$,July $=3$, October =4). This was carried out with the intention to simplify the study of the generated rules. Also, in order to simplify the process of classification, we proceeded to create a new variable called FANCLab indicating the status of the fan coil. This variable is defined by the values of temperature $\left({ }^{\circ} C\right)$ measured by sensor_ceiling (Fig.2). After processing these temperature values was generated three possible states of the fan coil ( $O$ is Off, 1 is Heating and 2 is Cooling).

\section{FUZZY LATTICE REASONING}

In this work, one of the goal is to define a intelligent system for decision making knowledge based on rules. Our idea is to compare different classifier and to show the advantage of fuzzy lattice reasoning classifier.

Fuzzy inference system is a technology based on fuzzy logic [13], that generates rules by induction process. Some previous work were presented hybrid system such as fuzzy lattice neurocomputing for clustering and classification in different data domains using lattice theory[14].

The fuzzy lattice reasoning classifier generates rules from the training data in two step rule induction and generalization[15]. Four important ideas of fuzzy lattice reasoning can be summarized[15]:

- The rule induction may be effected by replacing a hyperbox $A_{j}$ by a larger hyperbox $a_{i} \bigvee A_{j}$, where the larger hyperbox is assigned category label $C_{j}$.

- A rule $A_{j} \longrightarrow C_{j}, \mathrm{j}=1, \ldots, \mathrm{L}$ defines a fuzzy set $\mathrm{k}\left(\mathrm{x} \leq A_{j}\right)$ in the family of hyperboxes such that hyperbox $A_{j}$ corresponds to the core of fuzzy set $\mathrm{k}(\mathrm{x}$ $\leq A_{j}$ ).

- Fuzzy lattice reasoning can deal with semantic data and also non-numeric data, e.g. structured data (graphs).

- Fuzzy lattice reasoning classifier can deal with a missing data value in a constituent lattice $L_{i}$ by replacing a missing datum with a lattice interval $[\mathrm{a}, \mathrm{b}]$ such that $v_{i}([a, b])=v_{i}\left(\theta_{i}(a)\right)+v_{i}(b) \cong 0$. The latter replacement is semantically interpreted as absence of information.

More information about the full description of the induction and generalization algorithm can be found in[15]. The good accuracy rate and the simple generated rules are the two main reason for implemented this making decision system in CIESOL.

\section{EXPERIMENTAL RESULTS AND DISCUSSION}

Many classifiers were applied in our study about CIESOL. These classifiers were selected based on their low computational cost and good results reported in the literature. The selected classifiers are One R, C4.5, Naive Bayes, Multilayer Perceptron, K-nearest neighbor algorithm and NNge - genetic algorithm. The C4.5 algorithm [16] builds a decision tree 


\begin{tabular}{|c|c|c|}
\hline \multicolumn{3}{|c|}{ Variable Specification } \\
\hline Name & Description & Data type \\
\hline Ti_Sup_Lab & $\begin{array}{l}\text { Temperature maesured in } \\
\text { room }\left({ }^{\circ} C\right)\end{array}$ & Numeric \\
\hline AlarActivePres & Central alarm activated $(0,1)$ - Alarm active presence & Boolean \\
\hline SenMovLab & Motion sensor, $(0,1)$ & Boolean \\
\hline Month & $\begin{array}{l}\text { Month when the measurements } \\
\text { were made } \\
\text { (February - 1, May - 2, July - 3, November - 4) }\end{array}$ & Numeric \\
\hline FANCLab & Fancoil Output: 0 Off, 1 Heating and 2 Cooling & Numeric \\
\hline
\end{tabular}

using entropy. The Naive Bayes classifier [17] is the simplest bayesian network. It assumes that given the class, all variables are independent of each other. Multilayer Perceptron (MLP) [18] (artificial neural network) uses back-propagation to classify different actions of our system depending on the previous information. $K-N N$ ( $K$-nearest neighbors classifier) [19] assigns the action to its nearest neighbor's class. NNge[20] is based on genetic algorithm. Finally, the OneR classifier [21] uses the minimum-error attribute for prediction, discretizing numeric attributes. Ten-folds cross-validation was used for all tests.

To apply the classifiers previously described algorithms included in the platform Waikato Environment for Knowledge Analysis (WEKA) [22] version 3.5.8. have been used. The platform has been executed on an Intel (R) Core (TM) i7 processor, $2.6 \mathrm{GHz}$ and $16 \mathrm{~GB}$ of RAM computer. The results obtained after the application of the classifiers are shown in Table II.

\section{A. One $R$}

The results obtained by applying the classifier OneR were not satisfactory because two of the three generated rules were inconsistent. In addition to this, a high percentage of incorrectly classified instances $(29,341 \%)$ were obtained.

\section{B. Naive Bayes}

After applying the survey data, this classifier generated quite acceptable results. However the classifier does not generate any rules, since the results obtained are likelihood ratios of each of the nodes, thus there are no dependency relationships between attributes.

\section{C. $I B K$}

Although the time taken to build the model of the IBK classifier was the lowest of all classifiers, this classifier generated the highest rate of incorrectly classified instances $(31.8815 \%)$ with the highest mean absolute error $(0.2125 \%)$ amongst all classifiers applied.

\section{Multilayer Perceptron Neural Network}

Although the results obtained by applying the MultilayerPerceptron classifier [5] are quite satisfactory $(99.519 \%$ of instances classified correctly) the time taken by this classifier to generate the model is too high for the purpose of this study $(159.24 \mathrm{sec})$, therefore it was discarded.

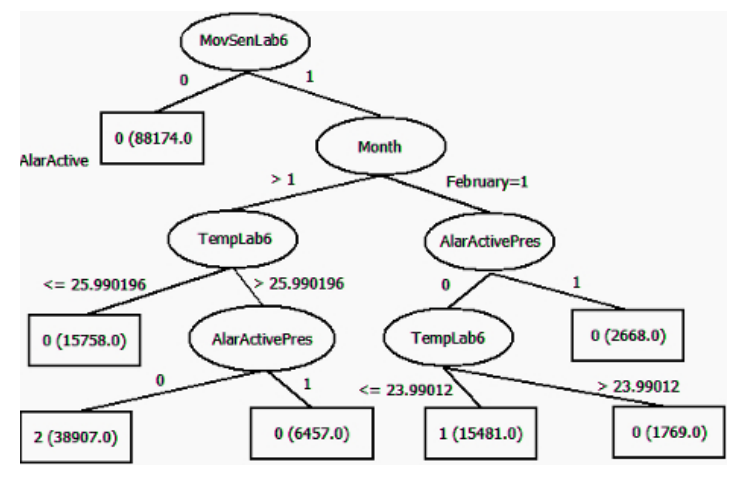

Fig. 5. Decision tree generated with C4.5 algorithm.

\section{E. NNge}

With the classifier NNge good results were achieved in both training time and classification $(96.4578 \%$ of instances classified correctly). From the six rules generated one of them was incongruous. The study of these rules shows that there is a reference temperature based on which, depending on the month of the year, the cooling or heating is turned on or off, and that this temperature varies depending on the room, yielding the following reference temperatures (comfort temperature) per room: Lab 6: Reference temperature $=25^{\circ} \pm 1^{\circ}$; Lab 8: Reference temperature $=24^{\circ} \pm 1^{\circ}$.

\section{F. C4.5 Decision Tree}

With the C4.5 classifier [7]it has been applied a Pruning Confidence Factor (CFP) of 0.25 , yielding a decision tree (DT) with a height of 13 and 7 sheets (rules) and with an accuracy of $96.6025 \%$ correctly classified instances. From the revision of the generated rules ,the reference temperatures observed in the rooms, that were generated by classifier NNge rules, are confirmed. Furthermore, it is observed that the heating is only turned on in the month of February (winter) while the air conditioning is only operational in non-winter months (May, July, October). In figure for (Fig. 5) the decision tree generated given the values of the laboratory 6 is shown.

\section{G. Fuzzy Lattice Reasoning}

The first results achieved by applying this classifier with RhoA $=0.5$ were unsatisfactory resulting in $27.1626 \%$ of instances incorrectly classified and a root mean squared error of 0.4255 . Nevertheless it was decided to continue using this classifier because it seemed to adjust to the nature of the problem at hand. So, after applying a 0.8 RhoA results improved significantly reducing the incorrectly classified instance 


\begin{tabular}{|c|c|c|c|c|c|c|c|c|c|}
\hline Classifier & $\begin{array}{l}\text { Time taken } \\
\text { To Build } \\
\text { Model(sg) }\end{array}$ & $\begin{array}{l}\text { Number } \\
\text { of } \\
\text { Rules }\end{array}$ & $\begin{array}{l}\text { Correctly } \\
\text { Classified } \\
\text { Instances }\end{array}$ & $\begin{array}{l}\text { Incorrectly } \\
\text { Classified } \\
\text { Instances }\end{array}$ & $\begin{array}{l}\text { Kappa } \\
\text { statistic }\end{array}$ & $\begin{array}{l}\text { Mean } \\
\text { absolute } \\
\text { error }\end{array}$ & $\begin{array}{l}\text { Root mean } \\
\text { squared } \\
\text { error }\end{array}$ & $\begin{array}{l}\text { Relative } \\
\text { absolute } \\
\text { error }\end{array}$ & $\begin{array}{l}\text { Root relative } \\
\text { squared } \\
\text { error }\end{array}$ \\
\hline FLR(rhoa 0.5) & 0.53 & 4 & 72.8374 & 27.1626 & 0.2329 & 0.1811 & 0.4255 & 56.117 & 105.941 \\
\hline FLR(rhoa 0.8 ) & 0.7 & 15 & 94.0673 & 5.9327 & 0.8705 & 0.0396 & 0.1989 & 12.2569 & 49.5117 \\
\hline FLR(rhoa 0.9) & 0.88 & 24 & 99.9976 & 0.0024 & 1 & 0 & 0.004 & 0.0049 & 0.9883 \\
\hline IBK & 0.02 & & 68.1185 & 31.8815 & 0.2663 & 0.2125 & 0.461 & 66.7205 & 115.6903 \\
\hline C4.5 & 1.34 & 7 & 96.6025 & 3.3975 & 0.9313 & 0.0428 & 0.1218 & 13.414 & 30.4927 \\
\hline MultilayerPerceptron & 159.24 & & 99.519 & 0.481 & 0.9901 & 0.004 & 0.0497 & 1.2277 & 12.3844 \\
\hline NaiveBayes & 0.38 & & 97.1492 & 2.8508 & 0.9426 & 0.0455 & 0.1196 & 14.1098 & 29.7878 \\
\hline NNge & 3.61 & 6 & 96.4578 & 3.5422 & 0.9287 & 0.0468 & 0.1244 & 14.5182 & 31.071 \\
\hline OneR & 0.47 & 3 & 70.659 & 29.341 & 0.3885 & 0.1956 & 0.4423 & 60.618 & 110.107 \\
\hline
\end{tabular}

TABLE II. RESULTS OBTAINED AFTER THE APPLICATION OF THE CLASSIFIERS

to 5.9327. This significant improvement in the results led to further implementation of the FLR classifier adjusting the value of RhoA so that finally, by assigning the FLR classifier with $\mathrm{RhoA}=0.9$ it was possible to achieve only one $0.0024 \%$ of incorrectly classified instances.

Therefore, applying the classifier FLR brought significant improvement over the results obtained by applying the classifier C4.5 (99.9976\% of instances classified Correctly and a $0.9883 \%$ of relative squared Root Error applying the FLR classifier was obtained, compared to $96.6025 \%$ and a $30.4927 \%$ respectively from applying the classifier $\mathrm{C} 4.5$ ).

These excellent results can be seen in the accuracy scatter plot after applying the FLR classifier contained in figure five (Fig. 6). In this graph it can be seen that incorrectly classified instances are not significant and that the rules generated are confirmed after applying the classifier C4.5, meaning there are reference temperatures that match after applying the two classifiers and the possible phase that the fan coil can be (heating, air conditioning) is determined by the months of the year (seasons).

While improved results of the $\mathrm{C} 4.5$ classifier compared to classifier FLR is generated in the number of rules (C4.5 classifier 7 vs 24 FLR) this aspect is not relevant, since the rules generated by the classifier FLR can be easily implemented thanks to its simplicity.

Below are 4 of the 24 generated rules:

- Rule 1: If $14.67 \leq$ TempLab6 $\leq 23.98$ and MovSenLab6 and Not AlarActivePres and Month $=1$ then Heating

- Rule 2: If $25.99 \leq$ TempLab6 $\leq 30.98$ and MovSenLab6 and Not AlarActivePres and Month $=3$ then Cooling

- Rule 3: If $25.99 \leq$ TempLab6 $\leq 30.98$ and Not MovSenLab6 and Not AlarActivePres and Month $=3$ then Off

- Rule 4: If $25.99 \leq$ TempLab6 $\leq 30.98$ and MovSenLab6 and AlarActivePres and Month $=3$ then Off

As is can be observe these rules concur with the rules obtained after applying the classifier $\mathrm{C} 4.5$ for both the reference

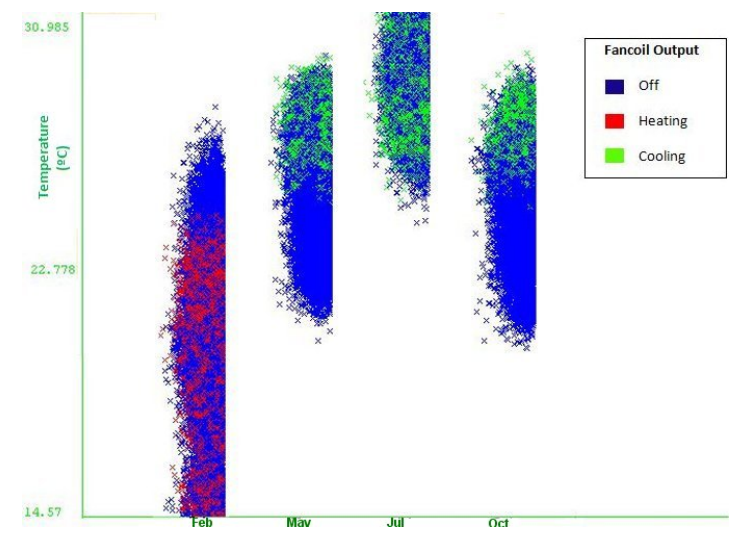

Fig. 6. Accuracy dispersion of FLR (rhoa 0.9) for 4 Months in 2014.

temperature $\left(25^{\circ} \pm 1^{\circ}\right)$ and the possible state of the fan coil depending on the month of the year.

In conclusion, we use the FLR classifier in our decisionmaking because this classifier improves results of the other classifiers shown in this paper. Moreover, the FLR classifier allowes to interprete qualitative, imprecise and uncertain data source such as our study case.

\section{CONCLUSION}

In this paper we have been successful in obtaining rules that define the fan coil's behavior in the rooms that have been object of study, based on the data compiled from several sensors in the CIESOL building during a year. These rules provide, on the one hand, an increase in the domotic system's energetic efficiency as they avoid the fan coil from remaining active while no users are present in the rooms.

On the other hand, they also avoid the fan coil from turning on and off due to programming that does not consider the comfort temperatures for users, even when temperatures inside those rooms have already reached the desired value for users.

Moreover, and more important, the rules obtained provide the domotic system with the ability to adapt to the user, learning from the preferences of the later regarding comfort temperature and controlling the system in order to maintain that temperature. The domotic system will therefore be able to modify the reference or cofort temperatures in case other users are in the rooms or the users change their preferences (comfort temperature). In short, the solution presented in this paper equips the domotic system of CIESOL with the ability 
to adapt to the behavior of the user integrating a learning system into the domotic system, achieving an improvement of the system's energetic efficiency and increasing user comfort by adapting the system to the preferences of the user, thus freeing the later from controlling the components involved in the system.

As future work we will develop the necessary process to store the transformation rules generated by the decision making system as adaptation rules for our adaptative system schema and we will also extend.

The CIESOL building domotic system's ability to adapt by learning to other parts of the system that would benefit from considering users preferences in order to achieve the before mentioned goals. We will specifically apply the domotic system's ability to adapt to control the indoor lighting, avoiding the system from switching the lights on and off based on certain preset light intensity values. Instead the system will adapt itself by learning, to the preferences of the users regarding light intensity.

\section{ACKNOWLEDGMENT}

This work was funded by the EU ERDF and the Spanish Ministry of Economy and Competitiveness (MINECO) under Project TIN2013-41576-R, and the Andalusian Regional Government (Spain) under Project P10-TIC-6114. This work also received funding from the $\mathrm{CEiA} 3$ and CEIMAR consortiums. We thank our colleagues from CIESOL and Solar Energy Resources and Climatology research group (TEP165), who provided data and expertise that greatly assisted the research.

\section{REFERENCES}

[1] CIESOL building, http://www.ciesol.es/en/content/ciesol-building.

[2] Criado Javier, Rodríguez-Gracia Diego, Iribarne Luis, and Padilla Nicolás, "Toward the adaptation of component-based architectures by model transformation: behind smart user interfaces", Softw. Pract. Exper, 2014

[3] Mihail-Bogdan Carutasiu, Vladimir Tanasiev, Constantin Ionescu, Alexandra Danu, Horia Necula, Adrian Badea, "Reducing energy consumption in low energy buildings through implementation of a policy system used in automated heating systems", Energy and Buildings, vol. 94, no. 1, pp. 227-239, 2015.

[4] Mavridis, Nikolaos and Pierris, Georgios and BenAbdelkader, Chiraz and Krstikj, Aleksandar and Karaiskos, Christos, "Smart buildings and the human-machine cloud", GCC Conference and Exhibition (GCCCE), 2015 IEEE 8th, pp. 1-6, Feb, 2015.

[5] T. A. Nguyen, M. Aiello, "Beyond Indoor Presence Monitoring with Simple Sensors", Proceedings of the 2nd International Conference on Pervasive and Embedded Computing and Communication Systems, 2012.

[6] Y. Agarwal, B. Balaji, R. Gupta, J. Lyles, M. Wei, T. Weng, "Occupancydriven energy management for smart building automation", Proceedings of the 2nd ACM Workshop on Embedded Sensing Systems for EnergyEfficiency in Building, BuildSys 10, ACM, New York, NY, USA, 2010, pp. $1-6,2010$

[7] A. Marchiori, Q. Han, "Distributed wireless control for building energy management", Proceedings of the 2nd ACM Workshop on Embedded Sensing Systems for Energy-Efficiency in Building, BuildSys 10, ACM, New York, NY, USA, 2010, pp. 37-42, 2010.

[8] C. Harris, V. Cahill, "Exploiting user behaviour for context-aware power management", Wireless And Mobile Computing, Networking And Communications, 2005. (WiMob2005), IEEE International Conference, vol. 4, pp. 122-130, 2005.
[9] L. Hawarah, S. Ploix, M. Jacomino, "User behavior prediction in energy consumption in housing using Bayesian networks", Proceedings of the 10th international conference on Artificial intelligence and soft computing: Part I, ICAISC10, Springer-Verlag, Berlin, Heidelberg, pp. 372-379, 2010.

[10] H. Hagras, V. Callaghan, M. Colley, G. Clarke, A. Pounds-Cornish, H. Duman, "Creating an ambient-intelligence environment using embedded agents", intelligent systems, IEEE, vol. 19, no. 6, pp. 12-20, 2004.

[11] R.H. Dodier, G.P. Henze, D.K. Tiller, X. Guo, "Building occupancy detection through sensor belief networks", Energy and Buildings , vol. 38, no. 9, pp. 1033-1043, 2006

[12] Tuan Anh Nguyen, Marco Aiello, "Energy intelligent buildings based on user activity: A survey", Energy and Buildings, vol. 56, pp. 244-257, 2013

[13] V.G. Kaburlasos, A. Kehagias, "Novel fuzzy inference system (FIS) analysis and design based on lattice theory", IEEE Transactions on Fuzzy Systems, vol. 15, no. 2, pp. 243-260,2007.

[14] V. Petridis, V. G. Kaburlasos, "Clustering and classification in structured data domains using Fuzzy Lattice Neurocomputing (FLN)", IEEE Transactions on Knowledge and Data Engineering vol. 13, no. 2, pp. 245-260, 2001.

[15] V. G. Kaburlasos, I. N. Athanasiadis, P. A. Mitkas, "Fuzzy lattice reasoning (FLR) classifier and its application for ambient ozone estimation", International Journal of Approximate Reasoning vol. 45, no. 1, pp. 152$188,2007$.

[16] J. R. Quinlan, C4.5: programs for machine learning. Morgan kaufmann, 1993, vol. 1.

[17] G. H. John and P. Langley, "Estimating continuous distributions in Bayesian classifiers," in Proceedings of the Eleventh conference on Uncertainty in artificial intelligence. Morgan Kaufmann Publishers Inc., 1995, pp. 338-345.

[18] M. L. Minsky and S. A. Papert, Perceptrons - Expanded Edition: An Introduction to Computational Geometry. MIT press Boston, MA:, 1987.

[19] D. W. Aha, D. Kibler, and M. K. Albert, "Instance-based learning algorithms," Machine learning, vol. 6, no. 1, pp. 37-66, 1991.

[20] B. Martin, "Instance-based learning: nearest neighbour with generalisation," Ph.D. dissertation, University of Waikato, 1995.

[21] R. C. Holte, "Very simple classification rules perform well on mos commonly used datasets," Machine learning, vol. 11, pp. 63-91, 1993.

[22] I. H. Witten, E. Frank, "Data Mining, Practical Machine Learning Tools and Techniques with Java Implementations," Morgan Kaufman Publishers, San Mateo, CA, 1999. 